\title{
Report of an abscopal effect induced by stereotactic body radiotherapy and nivolumab in a patient with metastatic non-small cell lung cancer
}

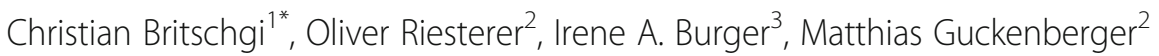 \\ and Alessandra Curioni-Fontecedro ${ }^{1}$
}

\begin{abstract}
Background: The existence of abscopal effects has been suggested already a long time ago, but only recently with the advent of immune checkpoint inhibition in clinical oncology and modern imaging techniques has it become possible to directly observe such effects in patients. They have been well described in patients with malignant melanoma being treated with immune-checkpoint inhibitors and stereotactic radiotherapy, but experience in other malignancies is very limited.

Case presentation: Here, we describe a case of a patient with metastatic non-small cell lung cancer, who experienced a complete response secondary to an abscopal effect on treatment with anti-PD-1 therapy and stereotactic body radiotherapy to some of the involved sites.

Conclusions: Our case reports confirms the existence of abscopal effects in NSCLC and suggests synergism between immune-checkpoint inhibition and local ablative RT. We suggest that this approach is now further studied in prospective clinical trials on oligo-metastatic or oligo-progressing NSCLC.
\end{abstract}

Keywords: Anti-PD-1 therapy, Nivolumab, Immune-checkpoint inhibition, Abscopal effect, Stereotactic body radiotherapy, Non-small cell lung cancer

\section{Background}

Mole introduced the term 'abscopal effect' in 1953 [1]. It describes a phenomenon characterized by tumor regression of untreated metastatic lesions after a local treatment, such as radiotherapy. This is thought to arise because ionizing irradiation causes localized cell death, which induces an immune response called immunogenic cell death. This is triggered by increased antigen release, by improved antigen presentation through increased expression of MHC I on the tumor cell surface, as well as by modulation of cytokines enhancing migration and function of effector CD8+ T cells [2]. However, this event is rare due to immunotolerance at the tumor site, leading to a reduced

\footnotetext{
* Correspondence: christian.britschgi@usz.ch

1 Department of Hematology and Oncology, University Hospital Zürich,

University of Zürich, Zürich, Switzerland

Full list of author information is available at the end of the article
}

systemic immune response. Treatment with immune checkpoint inhibitors might overcome tumor-related immunosuppression and start, as well as sustain the immune response towards cancer [3, 4].

\section{Case presentation}

We report on a 47-year-old male current smoker (40 $\mathrm{PY}$ ), who was diagnosed with lung adenocarcinoma (cT1a pN3 cM0, UICC Stage IIIB). He underwent combination treatment with chemotherapy and cetuximab, followed by radio-therapy in combination with cetuximab and surgical resection as part of a clinical trial (SAKK 16/08; NCT01059188). A pathologically complete response was achieved, but only 8 weeks post-operatively, retroperitoneal lymph node relapse occurred. Since sensitizing mutations were absent, we started palliative chemotherapy (cisplatin / pemetrexed, followed by pemetrexed maintenance).

(c) The Author(s). 2018 Open Access This article is distributed under the terms of the Creative Commons Attribution 4.0 International License (http://creativecommons.org/licenses/by/4.0/), which permits unrestricted use, distribution, and 
However, maintenance pemetrexed had to be discontinued after two cycles due to severe hematological side effects CTCAE grade 3, requiring in-patient treatment over several days.

After full recovery, a PET/CT scan performed four weeks after hospital demission revealed progressing abdominal lymph nodes (Fig. 1a). The patient was enrolled into an expanded access program (EAP) of the anti-programmed death 1 (PD-1) monoclonal antibody nivolumab. A first PET/CT scan after 6 cycles (i.e. 13 weeks after administration of the first nivolumab dose) showed a mixed response. The initially progressing sites were regressing, but three new abdominal lymph node metastases appeared (Fig. 1b).

The patient was treated with stereotactic body radiotherapy (SBRT) for this oligo-progression (Fig. 2a and b). Two out of the three lymph node metastases were irradiated $(3 \times 6 \mathrm{~Gy}=18 \mathrm{~Gy}$ at $80 \%$ isodose) (Figs. $1 \mathrm{~b}$ and $2 \mathrm{~b})$. The third lymph node remained un-irradiated because of close proximity to the small bowel and as reference lesion for immunotherapy. It received a radiation scatter dose of 0.4 Gy only, which is far below clinically significant anti-tumor doses. The patient continued treatment with nivolumab during SBRT and thereafter. A PET/CT scan 10 weeks after SBRT (after 13 nivolumab applications in total), showed a complete radiological and metabolic response (CR). Importantly, also the third lymph node metastasis, which had previously progressed and was not irradiated, showed a CR (Fig. 1c). Such a response after initial progression in the absence of any local treatment represents an abscopal effect provoked by PD-1 targeting in combination with SBRT.

During cycle 17, a severe pancreatitis CTCAE Grade 3 occurred and the patient had to be treated for several days as an in-patient, but eventually recovered fully. In the absence of other provoking factors, the most likely differential diagnosis was immune-related pancreatitis and nivolumab was therefore permanently stopped. The patient is today, almost two years after permanently stopping nivolumab, still in complete remission and in regular follow-up in our department (Fig. 1d).

\section{Discussion and conclusions}

Here we describe a case of a patient with metastatic NSCLC experiencing a complete response on treatment with anti-PD-1 therapy and SBRT. A biopsy of the non-irradiated lesion before SBRT was not clinically feasible. Therefore, we cannot principally rule out alternative explanations, such as for example a delayed response to immune checkpoint inhibition. However, the temporal course is highly suggestive of a true abscopal effect.

The existence of abscopal effects has been suggested already several decades ago, but only with the advent of immune-checkpoint inhibitors in clinical routine has it become possible to observe those effects directly in patients. There are several case reports and retrospective analyses suggesting that combining immune-checkpoint inhibition with SBRT might be beneficial in patients with

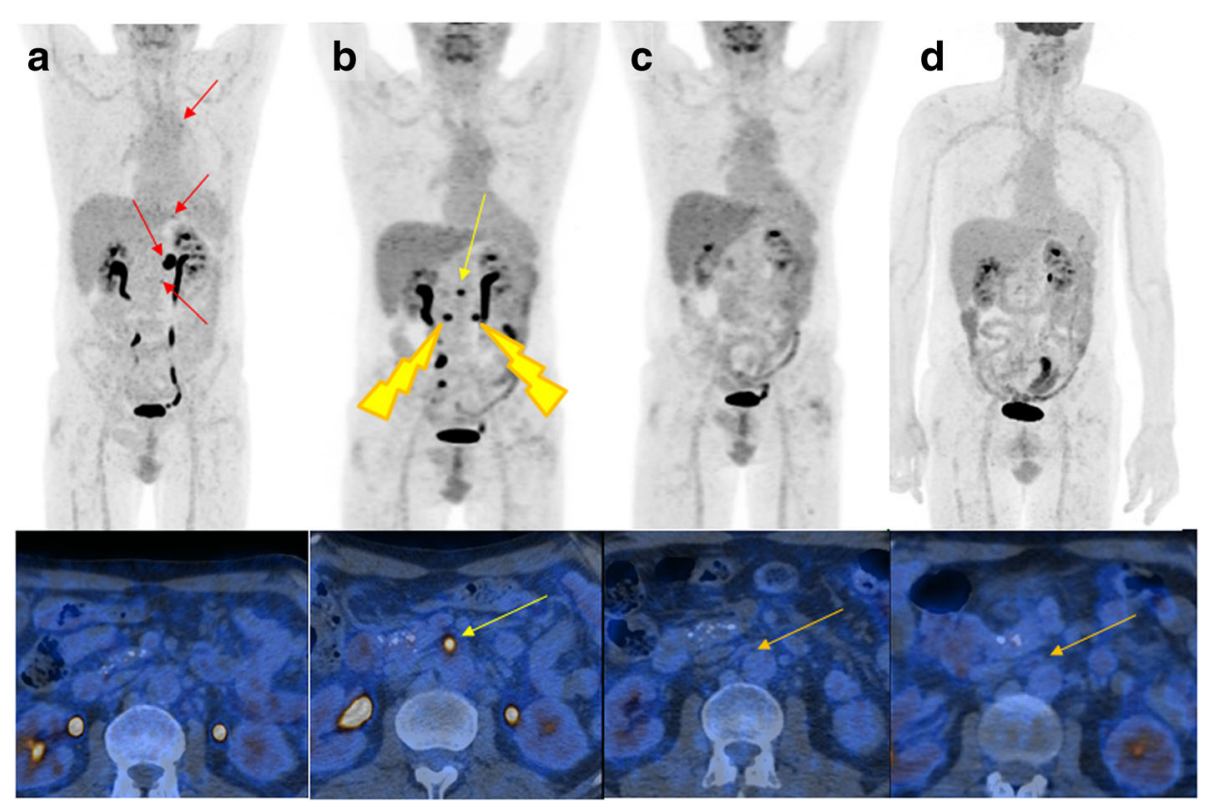

Fig. 1 a PET/CT staging before start of treatment with nivolumab: the red arrows indicate the localization of the lymph node metastases. $\mathbf{b}$ PET/CT re-staging after 6 cycles of nivolumab with evidence of complete response of the previous metastases and appearance of new metastases, indicated by yellow arrows. Two out of the three new metastases were irradiated, as indicated. c PET/CT restaging 10 weeks after radiotherapy with evidence of complete response. $\mathbf{d}$ PET/CT restaging two years after start of nivolumab confirming a stable complete remission 


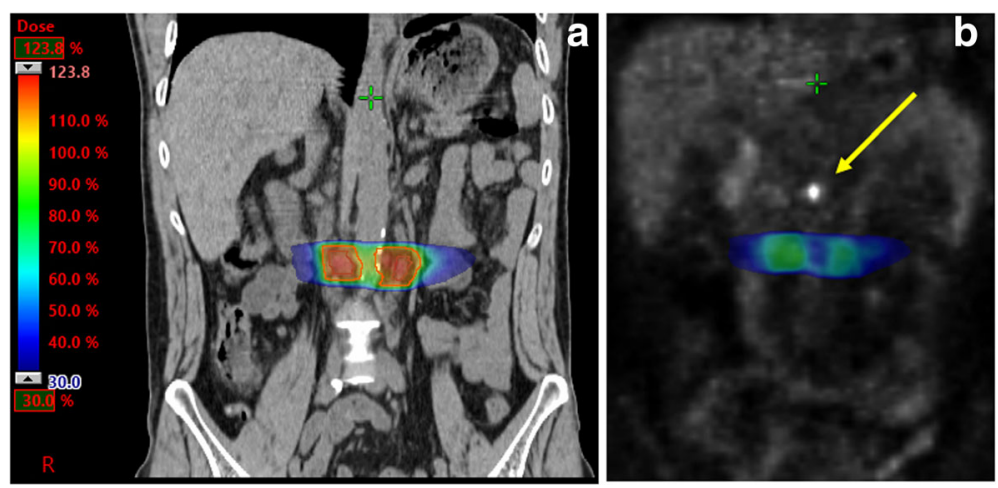

Fig. 2 a Coronal image of the dose distribution of radiotherapy. The patient received $3 \times 6$ Gy @ 80\%. b Image fusion of FDG-PET and treatment plan showing $30 \%$ of the prescribed dose (blue) in relation to the untreated FDG-positive lymph node (indicated by the yellow arrow)

malignant melanoma [5, 6]. In a first report of 101 patients treated with the anti-CTLA4 immune-checkpoint inhibitor ipilimumab, 70 received radiotherapy at some point during their treatment and 31 did not. The median overall survival (OS) in a retrospective analysis was significantly increased in the group, which received RT (19 months vs. 10 months for ipilimumab alone [ $p=0.01]$ ) [5]. A similar observation was made in a second analysis studying specifically patients who received anti-PD-1 immune-checkpoint inhibition and radiotherapy. Of 59 patients who received pembrolizumab $(n=28)$ or nivolumab $(n=31), 17$ also received palliative RT. The combination was not associated with increased toxicity and the objective response rate (complete or partial response) was significantly higher in the group, which had received RT (64.7 vs. $33.3 \%, P=0.02)$, including one complete responder who exhibited a classical abscopal effect. Such abscopal effects might be especially triggered by RT when limited to the involved region, as showed in preclinical models, in which spearing of draining lymph nodes is crucial to develop antitumor responses [7]. Taken together, these observations indicate that combining RT with immune-checkpoint inhibition (either targeting CTLA4 or PD-1) is well tolerated and has therapeutic potential in malignant melanoma.

Experience in other solid malignancies is more limited, given that immune-checkpoint inhibition first entered clinical routine in melanoma. There are some indications that abscopal effects also exist in NSCLC. A case report described a spontaneous regression of a second pulmonary lesion after having applied SBRT to a first lesion only [8], and there is also a report of an abscopal effect in a patient with adenocarcinoma of the lung receiving a combination of anti-CTLA4 inhibition (using ipilimumab) and SBRT [9]. Moreover, there is evidence from the randomized phase III trial PACIFIC that consolidation immune-checkpoint inhibition using durvalumab after concurrent, definite radio-chemotherapy is beneficial in patients with stage III NSCLC [10].

Our observation in this case now suggests a synergy of concurrent immune-checkpoint inhibition targeting the PD-1/PD-L1 axis and local ablative radiotherapy in NSCLC, as well. This approach should now be studied further in prospective clinical trials in the context of oligo-progressing and oligo-metastatic NSCLC.

\section{Abbreviations \\ CR: Complete response; NSCLC: Non-small cell lung cancer; PD-1: Programmed death-1; SBRT: Stereotactic body radiotherapy \\ Availability of data and materials \\ All data generated or analyzed during this study are included in this published article. The datasets used and/or analyzed during the current study are available from the corresponding author on reasonable request.}

\section{Authors' contributions}

CB and ACF analyzed and interpreted the patient's data and wrote the report, OR and MG performed the SBRT, IAB performed and analyzed the PET-CT scans. All authors edited, approved and read the manuscript.

Ethics approval and consent to participate

This report has been performed in accordance with the Declaration of Helsinki.

Consent for publication

The patient consented to publication of the data.

\section{Competing interests}

The authors declare that they have no competing interests.

\section{Publisher's Note}

Springer Nature remains neutral with regard to jurisdictional claims in published maps and institutional affiliations.

\section{Author details}

'Department of Hematology and Oncology, University Hospital Zürich, University of Zürich, Zürich, Switzerland. '2Department of Radiation Oncology, University Hospital Zürich, University of Zürich, Zürich, Switzerland.

${ }^{3}$ Department of Nuclear Medicine, University Hospital Zürich, University of Zürich, Zürich, Switzerland. 
Received: 25 March 2018 Accepted: 16 May 2018

Published online: 31 May 2018

\section{References}

1. Mole RH. Whole body irradiation; radiobiology or medicine? $\mathrm{Br} J$ Radiol. 1953;26:234-41.

2. Formenti SC, Demaria S. Systemic effects of local radiotherapy. Lancet Oncol. 2009;10:718-26.

3. Borghaei $H$, Brahmer JR, Horn L, et al. Nivolumab (nivo) vs docetaxel (doc) in patients (pts) with advanced NSCLC: CheckMate 017/057 2-y update and exploratory cytokine profile analyses. J Clin Oncol. 2016;34(15_suppl):9025-25.

4. Reynders K, Illidge T, Siva S, et al. The abscopal effect of local radiotherapy: using immunotherapy to make a rare event clinically relevant. Cancer Treat Rev. 2015;41:503-10.

5. Koller KM, Mackley HB, Liu J, et al. Improved survival and complete response rates in patients with advanced melanoma treated with concurrent ipilimumab and radiotherapy versus ipilimumab alone. Cancer Biol Ther. 2017;18:36-42

6. Aboudaram A, Modesto A, Chaltiel L, et al. Concurrent radiotherapy for patients with metastatic melanoma and receiving anti-programmed-death 1 therapy: a safe and effective combination. Melanoma Res. 2017;27:485-91.

7. Zhang X, Niedermann G. Abscopal effects with Hypofractionated schedules extending into the effector phase of the tumor-specific T-cell response. Int J Radiat Oncol Biol Phys. 2018;101:63-73.

8. Cong Y, Shen G, Wu S, Hao R. Abscopal regression following SABR for non-small-cell-lung cancer: a case report. Cancer Biol Ther. 2017:18:1-3.

9. Golden EB, Demaria S, Schiff PB, et al. An abscopal response to radiation and ipilimumab in a patient with metastatic non-small cell lung cancer. Cancer Immunol Res. 2013;1:365-72.

10. Antonia SJ, Villegas A, Daniel D, et al. Durvalumab after Chemoradiotherapy in stage III non-small-cell lung Cancer. N Engl J Med. 2017;377:1919-29.

\section{Ready to submit your research? Choose BMC and benefit from:}

- fast, convenient online submission

- thorough peer review by experienced researchers in your field

- rapid publication on acceptance

- support for research data, including large and complex data types

- gold Open Access which fosters wider collaboration and increased citations

- maximum visibility for your research: over $100 \mathrm{M}$ website views per year

At BMC, research is always in progress.

Learn more biomedcentral.com/submissions 